\title{
Learning to Automatically Generate Fill-In-The-Blank Quizzes
}

\author{
Edison Marrese-Taylor, Ai Nakajima, Yutaka Matsuo \\ Graduate School of Engineering \\ The University of Tokyo \\ emarrese, ainakajima, matsuo@weblab.t.u-tokyo.ac.jp
}

Ono Yuichi

Center for Education of Global Communication

University of Tsukuba

ono.yuichi.ga@u.tsukuba.ac.jp

\begin{abstract}
In this paper we formalize the problem automatic fill-in-the-blank question generation using two standard NLP machine learning schemes, proposing concrete deep learning models for each. We present an empirical study based on data obtained from a language learning platform showing that both of our proposed settings offer promising results.
\end{abstract}

\section{Introduction}

With the advent of the Web 2.0, regular users were able to share, remix and distribute content very easily. As a result of this process, the Web became a rich interconnected set of heterogeneous data sources. Being in a standard format, it is suitable for many tasks involving knowledge extraction and representation. For example, efforts have been made to design games with the purpose of semi-automating a wide range of knowledge transfer tasks, such as educational quizzes, by leveraging on this kind of data.

In particular, quizzes based on multiple choice questions (MCQs) have been proved efficient to judge students knowledge. However, manual construction of such questions often results a timeconsuming and labor-intensive task.

Fill-in-the-blank questions, where a sentence is given with one or more blanks in it, either with or without alternatives to fill in those blanks, have gained research attention recently. In this kind of question, as opposed to MCQs, there is no need to generate a WH style question derived from text. This means that the target sentence could simply be picked from a document on a corresponding topic of interest which results easier to automate.

Fill-in-the-blank questions in its multiplechoice answer version, often referred to as cloze questions (CQ), are commonly used for evaluating proficiency of language learners, including official tests such as TOEIC and TOEFL (Sakaguchi et al., 2013). They have also been used to test students knowledge of English in using the correct verbs (Sumita et al., 2005), prepositions (Lee and Seneff, 2007) and adjectives (Lin et al., 2007). Pino et al. (2008) and Smith et al. (2010) generated questions to evaluate students vocabulary.

The main problem in CQ generation is that it is generally not easy to come up with appropriate distractors -incorrect options- without rich experience. Existing approaches are mostly based on domain-specific templates, whose elaboration relies on experts. Lately, approaches based on discriminative methods, which rely on annotated training data, have also appeared. Ultimately, these settings prevent end-users from participating in the elaboration process, limiting the diversity and variation of quizzes that the system may offer.

In this work we formalize the problem of automatic fill-in-the-blank question generation and present an empirical study using deep learning models for it in the context of language learning. Our study is based on data obtained from our language learning platform (Nakajima and Tomimatsu, 2013; Ono and Nakajima; Ono et al., 2017) where users can create their own quizzes by utilizing freely available and open-licensed video content on the Web. In the platform, the automatic quiz creation currently relies on hand-crafted features and rules, making the process difficult to adapt. Our goal is to effectively provide an adaptive learning experience in terms of style and difficulty, and thus better serve users' needs (Lin et al., 2015). In this context, we study the ability of our proposed architectures in learning to generate quizzes based on data derived of the interaction of users with the platform. 


\section{Related Work}

The problem of fill-in-the-blank question generation has been studied in the past by several authors. Perhaps the earlies approach is by Sumita et al. (2005), who proposed a cloze question generation system which focuses on distractor generation using search engines to automatically measure English proficiency. In the same research line, we also find the work of Lee and Seneff (2007), Lin et al. (2007) and Pino et al. (2008). In this context, the work of Goto et al. (2009) probably represents the first effort in applying machine learning techniques for multiple-choice cloze question generation. The authors propose an approach that uses conditional random fields (Lafferty et al., 2001) based on hand-crafted features such as word POS tags.

More recent approaches also focus on the problem of distractor selection or generation but apply it to different domains. For example, Narendra and Agarwal (2013), present a system which adopts a semi-structured approach to generate CQs by making use of a knowledge base extracted from a Cricket portal. On the other hand, Lin et al. (2015) present a generic semi-automatic system for quiz generation using linked data and textual descriptions of RDF resources. The system seems to be the first that can be controlled by difficulty level. Authors tested it using an on-line dataset about wildlife provided by the BBC. Kumar et al. (2015) present an approach automatic for CQs generation for student self-assessment.

Finally, the work of Sakaguchi et al. (2013) presents a discriminative approach based on SVM classifiers for distractor generation and selection using a large-scale language learners corpus. The SVM classifier works at the word level and takes a sentence in which the target word appears, choosing a verb as the best distractor given the context. Again, the SVM is based on human-engineered features such as n-grams, lemmas and dependency tags.

Compared to approaches above, our take is different since we work on fill-in-the-blank question generation without multiple-choice answers. Therefore, our problem focuses on word selection - the word to blank - given a sentence, rather than on distractor generation. To the best of our knowledge, our system is also the first to use representation learning for this task.

\section{Proposed Approach}

We formalize the problem of automatic fill-on-theblanks quiz generation using two different perspectives. These are designed to match with specific machine learning schemes that are welldefined in the literature. In both cases. we consider a training corpus of $N$ pairs $\left(S_{n}, C_{n}\right), n=$ $1 \ldots N$ where $S_{n}=s_{1}, \ldots, s_{L\left(S_{n}\right)}$ is a sequence of $L\left(S_{n}\right)$ tokens and $C_{n} \in\left[1, L\left(S_{n}\right)\right]$ is an index that indicates the position that should be blanked inside $S_{n}$.

This setting allows us to train from examples of single blank-annotated sentences. In this way, in order to obtain a sentence with several blanks, multiple passes over the model are required. This approach works in a way analogous to humans, where blanks are provided one at a time.

\subsection{AQG as Sequence Labeling}

Firstly, we model the AQG as a sequence labeling problem. Formally, for an embedded input sequence $S_{n}=s_{1}, \ldots, s_{L(n)}$ we build the corresponding label sequence by simply creating a onehot vector of size $L\left(S_{n}\right)$ for the given class $C_{n}$. This vector can be seen as a sequence of binary classes, $Y_{n}=y_{1}, \ldots, y_{L(n)}$, where only one item (the one in position $C_{n}$ ) belongs to the positive class. Given this setting, the conditional probability of an output label is modeled as follows:

$$
\begin{array}{r}
p(y \mid s) \propto \prod_{i=1}^{n} \hat{y}_{i} \\
\hat{y}_{i}=H\left(y_{i-1}, y_{i}, s_{i}\right)
\end{array}
$$

Where, in our, case, function $H$ is modeled using a bidirectional LSTM (Hochreiter and Schmidhuber, 1997). Each predicted label distribution $\hat{y}_{t}$ is then calculated using the following formulas.

$$
\begin{array}{r}
\vec{h}_{i}=L S T M_{f w}\left(\vec{h}_{i-1}, x_{i}\right) \\
\overleftarrow{h_{i}}=\operatorname{LSTM} M_{b w}\left(\overleftarrow{h_{i+1}}, x_{i}\right) \\
\hat{y}_{i}=\operatorname{softmax}\left(\left[\vec{h}_{i} ; \overleftarrow{h_{i}}\right]\right)
\end{array}
$$

The loss function is the average cross entropy for the mini-batch. Figure 1 summarizes the proposed model.

$$
L(\theta)=-\frac{1}{n} \sum_{i=1}^{n} y_{i} \log \hat{y}_{i}+\left(1-y_{i}\right) \log \left(1-\hat{y}_{i}\right)
$$




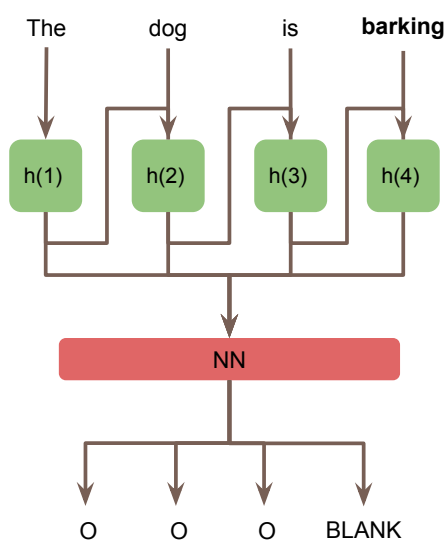

Figure 1: Our sequence labeling model based on an LSTM for AQG.

\subsection{AQG as Sequence Classification}

In this case, since the output of the model is a position in the input sequence $S_{n}$, the size of output dictionary for $C_{n}$ is variable and depends on $S_{n}$. Regular sequence classification models use a softmax distribution over a fixed output dictionary to compute $p\left(C_{n} \mid S_{n}\right)$ and therefore are not suitable for our case. Therefore, we propose to use an attention-based approach that allows us to have a variable size dictionary for the output softmax, in a way akin to Pointer Networks (Vinyals et al., 2015). More formally, given an embedded input vector sequence $S_{n}=s_{1}, \ldots, s_{L(n)}$, we use a bidirectional LSTM to first obtain a dense representation of each input token.

$$
\begin{array}{r}
\vec{h}_{i}=L S T M_{f w}\left(\vec{h}_{i-1}, x_{i}\right) \\
\overleftarrow{h}_{i}=\overleftarrow{L} S T M_{b w}\left(\overleftarrow{h}_{i+1}, x_{i}\right) \\
h_{i}=\left[\vec{h}_{i} ; \overleftarrow{h}_{i}\right]
\end{array}
$$

We later use pooling techniques including $\max$ and mean to obtain a summarized representation $\bar{h}$ of the input sequence, or simply take the last hidden state as a drop-in replacement to do so. After this, we add a global content-based attention layer, which we use to to compare that summarized vector to each hidden state $h_{i}$. Concretely,

$$
\begin{array}{r}
u=v^{\top} W\left[h_{i} ; \bar{h}\right] \\
p\left(C_{n} \mid P_{n}\right)=\operatorname{softmax}(u)
\end{array}
$$

Where $W$ and $v$ are learnable parameters of the model, and the softmax normalizes the vector $u$ to be an output distribution over a dictionary of size $L\left(S_{n}\right)$. Figure 2 summarizes the proposed model graphically. Then, for a given sentence $C_{k}$, the goal of our model is to predict the most likely position $C^{\star} \in\left[1, L\left(S_{n}\right)\right]$ of the next word to be blanked.

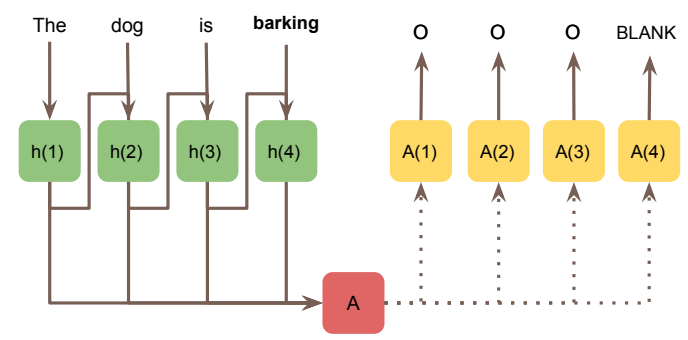

Figure 2: Our sequence classification model, based on an LSTM for AQG.

\section{Empirical Study}

Although the hand-crafted rule-based system currently used in our language learning platform offers us good results in general, we are interested in developing a more flexible approach that is easier to tailor depending on the case. In particular, in an adaptive learning setting where the goal is resource allocation according to the unique needs of each learner, rule-based methods for AQG appear to have insufficient flexibility and adaptability to accurately model the features of each learner or teacher.

With this point in mind, this section presents an empirical study using state-of-the-art Deep Learning approaches for the problem of AQG. In particular, the objective is to test to what extent our prosed models are able to encode the behavior of the rule-based system. Ultimately, we hope that these can be used for a smooth transition from the current human-engineered feature-based system to a fully user-experience-based regime.

In Natural Language Processing, deep models have succeeded in large part because they learn and use their own continuous numeric representational systems for words and sentences. In particular, distributed representations (Hinton, 1984) applied to words (Mikolov et al., 2013) have meant a major breakthrough. All our models start with random word embeddings, we leave the usage of other pre-trained vectors for future work.

Using our platform, we extracted anonymized user interaction data in the manner of real quizzes generated for a collection of several input video sources. We obtained a corpus of approximately 
300,000 sentences, from which roughly 1.5 million single-quiz question training examples were derived. We split this dataset using the regular 70/10/20 partition for training, validation and testing.

As the system required the input sentences to be tokenized and makes use of features such as word pos-tags and such, the sentences in our dataset are processed using CoreNLP (Manning et al., 2014). We also extract user-specific and quiz-specific information, including word-level learning records of the user, such as the number of times the learner made a mistake on that word, or whether the learner looked up the word in the dictionary. In this study, however, we restrain our model to only look at word embeddings as input.

We use the same data pre-processing for all of our models. We build the vocabulary using the train partition of our dataset with a minimum frequency of 1 . We do not keep cases and obtain an unknown vocabulary of size 2,029 , and a total vocabulary size of 66,431 tokens.

\subsection{Sequence Labeling}

We use a 2-layer bidirectional LSTM, which we train using Adam Kingma and Ba (2014) with a learning rate of 0.001 , clipping the gradient of our parameters to a maximum norm of 5 . We use a word embedding size and hidden state size of 300 and add dropout (Srivastava et al., 2014) before and after the LSTM, using a drop probability of 0.2. We train our model for up to 10 epochs. Training lasts for about 3 hours.

For evaluation, as accuracy would be extremely unbalanced given the nature of the blanking scheme - there is only one positive-class example on each sentence- we use Precision, Recall and F1-Score over the positive class for development and evaluation. Table 1 summarizes our obtained results.

\begin{tabular}{c|c|c|c|c} 
Set & Loss & Prec. & Recall & F1-Score \\
\hline Valid & 0.0037 & 88.35 & 88.81 & 88.58 \\
Test & 0.0037 & 88.56 & 88.34 & 88.80
\end{tabular}

Table 1: Results of the seq. labeling approach.

\subsection{Sequence Classification}

In this case, we again use use a 2-layer bidirectional LSTM, which we train using Adam with a learning rate of 0.001 , also clipping the gradient of our parameters to a maximum norm of 5. Even with these limits, convergence is faster than in the previous model, so we only trained the the classifier for up to 5 epochs. Again we use a word embedding and hidden state of 300 , and add dropout with drop probability of 0.2 before and after the LSTM. Our results for different pooling strategies showed no noticeable performance difference in preliminary experiments, so we report results using the last hidden state.

For development and evaluation we used accuracy over the validation and test set, respectively. Table 2 below summarizes our obtained result, we can see that model was able to obtain a maximum accuracy of approximately $89 \%$ on the validation and testing sets.

\begin{tabular}{c|c|c} 
Set & Loss & Accuracy \\
\hline Valid & 101.80 & 89.17 \\
Test & 102.30 & 89.31
\end{tabular}

Table 2: Results of the seq. classification approach.

\section{Conclusions}

In this paper we have formalized the problem of automatic fill-on-the-blanks quiz generation using two well-defined learning schemes: sequence classification and sequence labeling. We have also proposed concrete architectures based on LSTMs to tackle the problem in both cases.

We have presented an empirical study in which we test the proposed architectures in the context of a language learning platform. Our results show that both the 0 proposed training schemes seem to offer fairly good results, with an Accuracy/F1score of nearly $90 \%$. We think this sets a clear future research direction, showing that it is possible to transition from a heavily hand-crafted approach for AQG to a learning-based approach on the base of examples derived from the platform on unlabeled data. This is specially important in the context of adaptive learning, where the goal is to effectively provide an tailored and flexible experience in terms of style and difficulty

For future work, we would like to use different pre-trained word embeddings as well as other features derived from the input sentence to further improve our results. We would also like to test the power of the models in capturing different quiz styles from real questions created by professors. 


\section{References}

Takuya Goto, Tomoko Kojiri, Toyohide Watanabe, Tomoharu Iwata, and Takeshi Yamada. 2009. An automatic generation of multiple-choice cloze questions based on statistical learning. In Proceedings of the 17th International Conference on Computers in Education, pages 415-422. Asia-Pacific Society for Computers in Education.

Geoffrey E Hinton. 1984. Distributed representations.

Sepp Hochreiter and Jürgen Schmidhuber. 1997. Long short-term memory. Neural computation, 9(8):1735-1780.

Diederik P. Kingma and Jimmy Ba. 2014. Adam: A method for stochastic optimization. CoRR.

G. Kumar, R. E. Banchs, and L. F. D'Haro. 2015. Automatic fill-the-blank question generator for student self-assessment. In 2015 IEEE Frontiers in Education Conference (FIE), pages 1-3.

John D. Lafferty, Andrew McCallum, and Fernando C. N. Pereira. 2001. Conditional Random Fields: Probabilistic Models for Segmenting and Labeling Sequence Data. In Proceedings of the Eighteenth International Conference on Machine Learning, ICML '01, pages 282-289, San Francisco, CA, USA. Morgan Kaufmann Publishers Inc.

John Lee and Stephanie Seneff. 2007. Automatic generation of cloze items for prepositions. In Eighth Annual Conference of the International Speech Communication Association.

Chenghua Lin, Dong Liu, Wei Pang, and Zhe Wang. 2015. Sherlock: A Semi-automatic Framework for Quiz Generation Using a Hybrid Semantic Similarity Measure. Cognitive Computation, 7(6):667-679.

Yi-Chien Lin, Li-Chun Sung, and Meng Chang Chen. 2007. An automatic multiple-choice question generation scheme for english adjective understanding. In Workshop on Modeling, Management and Generation of Problems/Questions in eLearning, the 15th International Conference on Computers in Education (ICCE 2007), pages 137-142.

Christopher D. Manning, Mihai Surdeanu, John Bauer, Jenny Finkel, Steven J. Bethard, and David McClosky. 2014. The Stanford CoreNLP natural language processing toolkit. In Association for Computational Linguistics (ACL) System Demonstrations.

Tomas Mikolov, Ilya Sutskever, Kai Chen, Greg S Corrado, and Jeff Dean. 2013. Distributed Representations of Words and Phrases and their Compositionality. In Advances in Neural Information Processing Systems 26. Curran Associates, Inc.

Ai Nakajima and Kiyoshi Tomimatsu. 2013. New potential of e-learning by re-utilizing open content online. In International Conference on Human Interface and the Management of Information.
Annamaneni Narendra and Manish Agarwal. 2013. Automatic cloze-questions generation. In Proceedings of the International Conference Recent Advances in Natural Language Processing RANLP 2013, pages 511-515.

Yuichi Ono and Ai Nakajima. Automatic quiz generator and use of open educational web videos for english as general academic purpose. In Proceedings of the 23rd International Conference on Computers in Education, pages 559-568. Asia-Pacific Society for Computers in Education.

Yuichi Ono, Ai Nakajima, and Manabu Ishihara. 2017. Motivational effects of a game-based automatic quiz generator using online educational resources for japanese efl learners. In Society for Information Technology and Teacher Education International Conference.

Juan Pino, Michael Heilman, and Maxine Eskenazi. 2008. A selection strategy to improve cloze question quality. In Proceedings of the Workshop on Intelligent Tutoring Systems for Ill-Defined Domains. 9th International Conference on Intelligent Tutoring Systems, Montreal, Canada, pages 22-32.

Keisuke Sakaguchi, Yuki Arase, and Mamoru Komachi. 2013. Discriminative approach to fill-in-theblank quiz generation for language learners. In Proceedings of the 51st Annual Meeting of the Association for Computational Linguistics (Volume 2: Short Papers), volume 2, pages 238-242.

Simon Smith, P. V. S. Avinesh, and Adam Kilgarriff. 2010. Gap-fill Tests for Language Learners: Corpus-Driven Item Generation.

Nitish Srivastava, Geoffrey Hinton, Alex Krizhevsky, Ilya Sutskever, and Ruslan Salakhutdinov. 2014. Dropout: A simple way to prevent neural networks from overfitting. The Journal of Machine Learning Research, 15(1):1929-1958.

Eiichiro Sumita, Fumiaki Sugaya, and Seiichi Yamamoto. 2005. Measuring Non-native Speakers' Proficiency of English by Using a Test with Automatically-generated Fill-in-the-blank Questions. In Proceedings of the Second Workshop on Building Educational Applications Using NLP, EdAppsNLP 05, pages 61-68, Stroudsburg, PA, USA. Association for Computational Linguistics.

Oriol Vinyals, Meire Fortunato, and Navdeep Jaitly. 2015. Pointer networks. In Advances in Neural Information Processing Systems, pages 2692-2700. 